Dept. of Virology,

Faculty of Veterinary Medicine, Beni-Suef University.

\title{
EMBRYO VACCINATION OF SPF EMBRYONATED CHICKEN EGGS WITH IBDV ALONE OR IN COMBINATION WITH NIGELLA SATIVA OIL
}

(With 2 Tables and 5 Figures)

By

\section{A.S. HUSSEIN; A.M. GALAL* and H. EL-ASKARY* \\ *Faculty of Pharmacy, Cairo University.}

(Received at 13/9/2008)

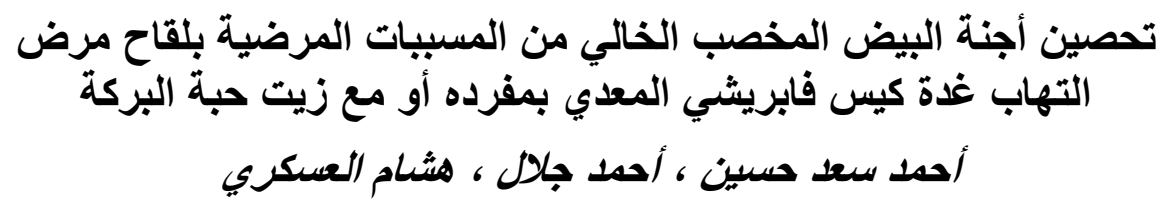

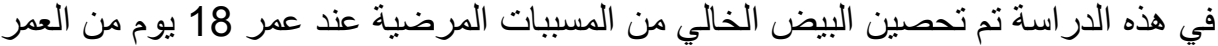

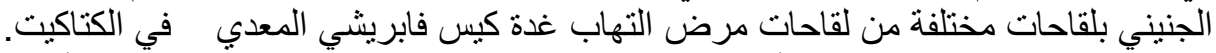

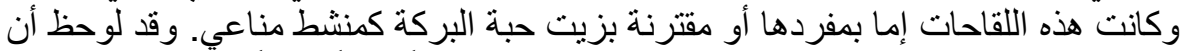

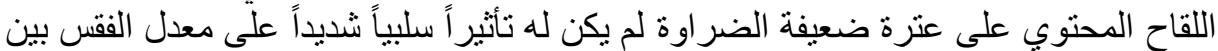

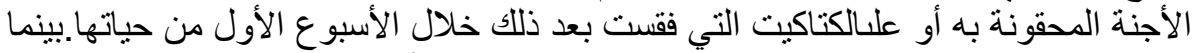

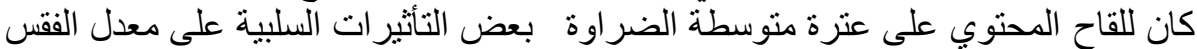

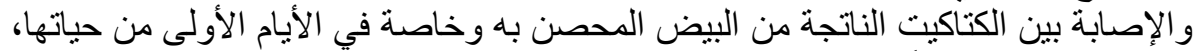

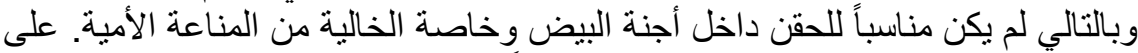

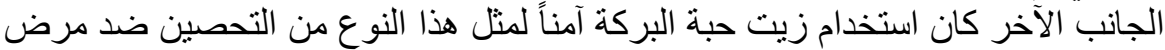

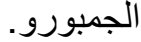

\section{SUMMARY}

In this study, specific-pathogen free chickens as embryos at the $18^{\text {th }}$ day of embryonation were vaccinated with infectious bursal disease (IBD) vaccine viruses of low and of moderate virulence either alone or in combination with Nigella sativa oil. The vaccine of low virulence did not adversely affect hatchability of chicks or survival of hatched chicks and developed antibody against IBDV. On the other hand, the vaccine virus of moderate virulence was found to be not suitable for use in SPFembryos (that free from maternal antibodies) as it caused extensive bursal damage in chickens of embryo vaccinated group. The IBD vaccines, especially of low virulence nature, could be combined with Nigella sativa oil and safely used as embryo-vaccination vaccine.

Key words: Infectious bursal disease, Gumboro, chicken, vaccination. 


\section{INTRODUCTION}

Infectious bursal disease (IBD), also known as Gumboro disease, is an acute, contagious viral disease of chickens. The disease causes severe immunosuppression and mortality in chickens of three to six weeks of age (Lukert and Saif, 1997). Infectious bursal disease virus (IBDV) targets the lymphoid tissue, specially the B-lymphocytes of the bursa of Fabricius (BF). In younger chickens, less than three weeks of age, IBDV causes subclinical disease with severe bursal damage, which leads to immunosuppression resulting in increased susceptibility to other infections, and vaccination failure (Kibenge et al., 1988). Thus, IBD is of major economic importance to the poultry industry. The protection of young chicks against IBD is an integral part of health management of most commercial poultry flocks.

Broiler breeders are immunized with live and inactivated vaccines in order to confer passive immunity to the progeny (Van den Berg et al., 1991).

During the first few weeks of life, broiler chicks are protected against IBDV by maternally acquired passive immunity. However, passive immunity decreases rapidly as the chick ages, leaving it susceptible to IBDV infection. Thus, it is a routine practice in the poultry industry to vaccinate young chicks against IBDV to control the disease. The optimal age for live vaccination in broilers is difficult to be predicted (Lutticken et al., 1994). If the vaccine is administered too early, neutralization of maternal antibodies may occur. Vaccine administration later in life may leave the birds susceptible to the disease. Unfortunately, despite these vaccination measures, IBDV continues to be a problem. Despite different vaccination programs the very virulent strains of IBDV, have caused outbreaks of disease with high mortality in Europe, Asia and all over the world (Nunoya et al., 1992; Van den Berg et al., 1991).

The development of new treatment techniques allows the application of pharmaceuticals and vaccines in ovo. The in ovo technology was initially developed for the application of Marek's vaccine in 18-day-old embryos (Sharma and Burmester, 1982). Today, this technology is also used for antibiotic therapy, delivery of other vaccines besides Marek's, egg-candling to determine viable fertile eggs, and transfer from incubators to hatchers. Also, in ovo technologies are being used to provide samples for diagnosis to help disease surveillance and epidemiological studies. The questions of major concern are the safety of the in ovo application route and the efficacy of the products 
applied via in ovo compared to the classical application routes (Jungbach et al., 1997).

Medicinal plants such as Nigella sativa,Fenugreek and Thymus valgaris, are economically important. Now days, there is an increase demand for using these herbal plants in therapy instead of synthetic drugs, which may have adverse effects (Mandour et al., 1998). Nigella sativa seeds is one of the most popular plants used in this field. The composition and properties of Nigella sativa seeds (black cumin) have been fairly investigated and the results of these investigations were reviewed (Houghton et al., 1995; EL-Alfy et al., 1975; Mahfouz and EL-Dakhakhny, 1960). These researchers reported that Nigella sativa seeds, or their extracts have anti-microbial, anti-histaminic, anti-tumour, anti- adhypertensive, anti-inflammatory and immuno-stimulant effects

The specific objective of this study is to evaluate the immunogenicity and protective efficacy of an in ovo IBDV vaccine plus Nigella sativa in SPF embryonated chicken eggs.

\section{MATERIALS and METHODS}

1- Embyonated chicken eggs: Experimental embyonated chicken eggs were obtained from specific-pathogen free parents of Kom Oshim Project; Fayum; Egypt. The eggs were incubated for 18 days in clean incubators and then injected with IBDV vaccines. The vaccine-injected eggs were hatched in isolators supplied with biologically filtered air.

2- Viruses and vaccines:

a- Mild strain of IBDV was derived from a commercial vaccine. Gumboral CT, Rhone Merieux 17 rue Bourgelat 69002 Lyon France. The virus was propagated in embryonated chcken eggs and stored at $20^{\circ} \mathrm{C}$. The virus stock has $50 \%$ embryo lethal dose $\left(\mathrm{ELD}_{50}\right) / \mathrm{ml}$ titer of $10^{4.7}$.

b- Moderately virulent isolate of IBDV was obtained from a commercial vaccine. Gumbokal, Bath No. 5042044, Manufacture for Pfizer Animal Health Group by Veterinary Ltd. Croatia, 70004715.

c- Challenge; The bursal homogenates of IBD local field isolate was obtained from Virology Department, Animal Health Research Institute, Dokki, Giza, Egypt. It was propagated in emryonated chicken eggs and had a titer of $10^{4.8} \mathrm{ELD}_{50} / \mathrm{ml}$.

3- Oil and emulsifiers:

- Nigella Sativa oil (whole crude oil): It was prepared from the Nigella Sativa seeds (commercial source) by pressing the seeds. 
- Sorbitan mono-oleate (SPAN 80): It is oil-soluble surfactant and was kindly obtained from Veterinary Serum and Vaccine Research Institute, Abbassia, Cairo,

- Polyoxy Ethylene Sorbitan (Tween 80): Non-ionic detergent was used as emulsifying agent to decrease the viscosity of the prepared vaccines.

4- Preparation of vaccines batch for in-ovo inoculation:

One part of the aqueous-phase (virus) was mixed with one part of the oil-phase (Nigella sativa oil). They were prepared with both the oilsoluble surfactant (SPAN 80) that added to the oil phase and aqueoussoluble surfactant (Tween 80) that added to the aqueous phase then both phases were emulsified manually over ten minutes to give homogenized emulsiom. Two types of live in oil-emulsion IBDV vaccines were prepared using Nigella Sativa oil as follows:

Vaccine (A) consisted of Gumboral vaccine (aqueous phase) + crud oil of N.sativa (oil phase).

Vaccine (B) consisted of Gumbokal vaccine (aqueous phase) + crud oil of N.sativa (oil phase).

The prepared vaccines were examined for the absence of aerobic and anaerobic bacteria, fungal and mycoplasma contaminants for being sterile.

5- Vaccination procedures:

Chickens were vaccinated as 18-day embryos with both vaccines (A and B) in addition to vaccine control (live vaccines without Nigella sativa oil) according to the routine procedures as described previously by Sharma (1985). The vaccines were applied to SPF chicken embryo in the chorio-allantoic membrane, with $0.1 \mathrm{ml}$ of inoculums using a $1.25 \mathrm{in}-$ long 22 gauge needle. After inoculation, the vaccinated eggs were returned to the incubator and allowed to hatch.

The detailed experimental protocol is shown in Table (1).

Table 1: Experimental protocol:

\begin{tabular}{|c|c|c|c|c|c|c|}
\hline Group & 1 & & 3 & 4 & 5 & 6 \\
\hline & \multicolumn{2}{|c|}{ Prepared vaccines } & \multicolumn{2}{|c|}{ Vaccine control } & Oil control & Negative control \\
\hline Treatment & $\begin{array}{l}\text { Vaccine (A) } \\
\text { Gumboral } \\
+ \text { crud oil of } \\
\text { N.sativa }\end{array}$ & $\begin{array}{l}\text { Vaccine (B) } \\
\text { Gumbokal } \\
\text { vaccine }+ \\
\text { crud oil of } \\
\text { N.sativa }\end{array}$ & $\begin{array}{l}\text { Gumboral } \\
\text { vaccine } \\
\text { only }\end{array}$ & $\begin{array}{l}\text { Gumbokal } \\
\text { vaccine } \\
\text { only }\end{array}$ & \begin{tabular}{|l} 
crud \\
oil of \\
Nigella \\
sativa
\end{tabular} & $\begin{array}{l}\text { One group } \\
\text { of the } \\
\text { chicken } \\
\text { embryos } \\
\text { was left } \\
\text { uninoculated }\end{array}$ \\
\hline $\begin{array}{l}\text { Number of } \\
\text { inoculated } \\
\text { chicken } \\
\text { embryos }\end{array}$ & 30 & 30 & 30 & 30 & 30 & 30 \\
\hline
\end{tabular}


The inoculated and un-inoculated chicken embryos were compared for hatchability and the hatched chicks were observed for clinical signs of IBD for one week after hatching.

6- Antibody response: The IBDV antibody titers of all vaccinated chickens and some unvaccinated control chickens were tested by ELISA at 7,14 and 21-days of age.

IBD ELISA kits: ELISA kits were obtained from IDEXX Laboratories and the ELISA test was carried out according to the manufacture instructions.

7- Challenge procedures:

The IBDV-vaccinated chickens and appropriate unvaccinated controls were challenged intra-ocularly at three weeks of age with virulent IBDV and observed for 7 days. The dying before the end of observation period and all survivors at the end of observation period were necropsied and examined for gross and examined for gross and microscopic lesions of IBD. The mean antibody titers at one-week post challenge of chickens vaccinated with different types of vaccines were detected.

\section{RESULTS}

Table 2: Effect of embryo inoculation with various treatment on hatchability and first week mortality of hatched chicks:

\begin{tabular}{|c|c|c|c|c|}
\hline \multirow[t]{2}{*}{ Group } & \multirow[t]{2}{*}{ Treatment } & \multicolumn{2}{|c|}{ Hatchability } & \multirow{2}{*}{$\begin{array}{l}\text { Mortality } \\
\text { within week } \\
\text { of hatch }\end{array}$} \\
\hline & & Hatched/inoculated & $\%$ hatchability & \\
\hline 1 & $\begin{array}{l}\text { Vaccine }(A) \\
\text { Gumboral + crud oil } \\
\text { of N.sativa }\end{array}$ & $24 / 30$ & $80 \%$ & 4.16 \\
\hline 2 & $\begin{array}{l}\text { Vaccine (B) } \\
\text { Gumbokal vaccine }+ \\
\text { crud oil of N.sativa }\end{array}$ & $16 / 30$ & 53.3 & 25 \\
\hline 3 & $\begin{array}{l}\text { Gumboral vaccine } \\
\text { Only }\end{array}$ & $26 / 30$ & 86.66 & 0 \\
\hline 4 & $\begin{array}{l}\text { Gumbokal vaccine } \\
\text { Only }\end{array}$ & $18 / 30$ & 60 & 22.22 \\
\hline 5 & $\begin{array}{l}\text { crud oil of Nigella } \\
\text { sativa }\end{array}$ & $25 / 30$ & 83.33 & 4 \\
\hline 6 & Negative control & $29 / 30$ & 96.6 & 0 \\
\hline
\end{tabular}


Fig. 1: Mean Antibody ELISA titers of serum at different interval of chickens vaccinated with different types of vaccines:

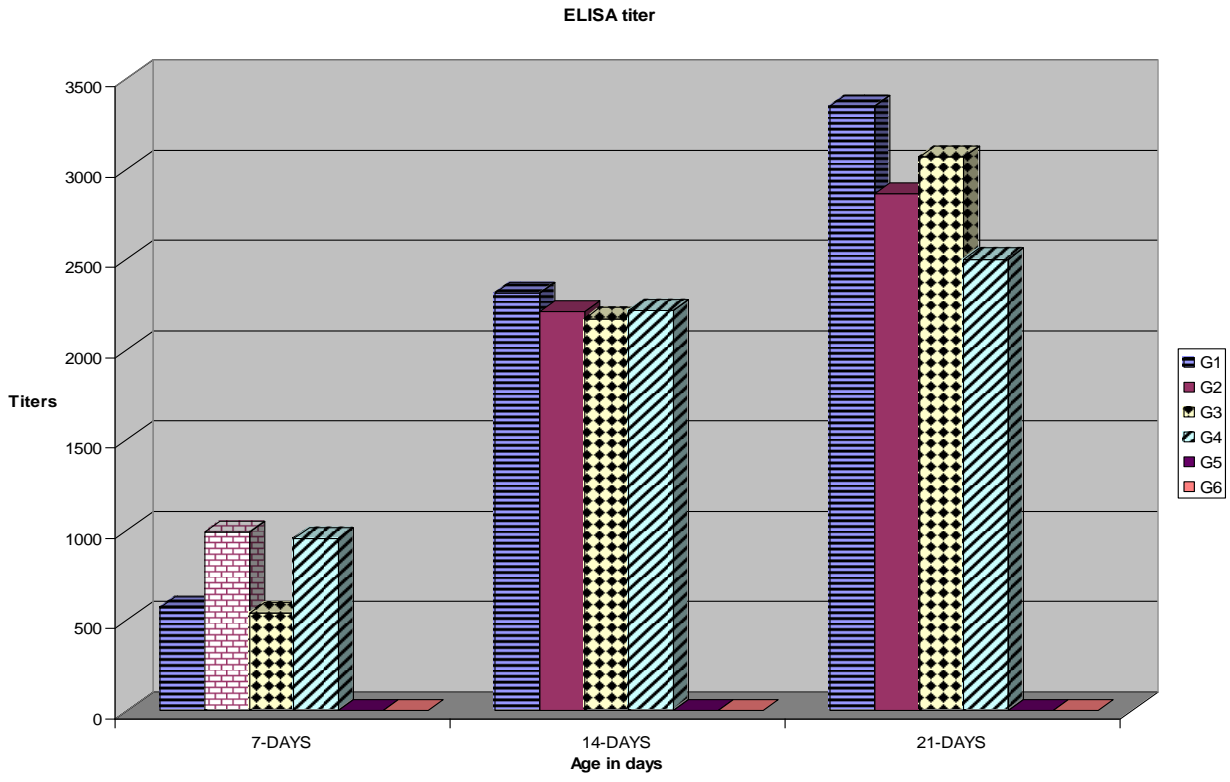

\section{Lesions response in chickens inoculated as embryos with IBDV vaccines:}

The eggs which not hatched and chicks that died within first week post-hatching were examined for bursal lesions. Results of this trial revealed that both embryo-vaccinated and hatched chicks had lesions, although these lesions were more pronounced in groups that vaccinated with vaccine virus of moderate virulence. The lesions in groups vaccinated with vaccine virus of low virulence were mild and tended to decrease in incidence and severity with time. The gross lesions included necrosis and atrophy of the bursa. Affected bursas had thin walls and appeared grayish. Microscopic lesions were characterized by acute inflammation accompanied by extensive lymphoid cell destruction, cyst formation, and hypertrophy of the inter-follicular connective tissue. In addition, no significant macroscopic or microscopic bursal lesions were observed in group that inoculated with Nigella sativa oil alone or in the control negative group. 
Fig. 2: Protection against IBDV in chickens vaccinated with different vaccines as 18-days embryos and challenged intra-ocularly at three weeks of age:

protection \%

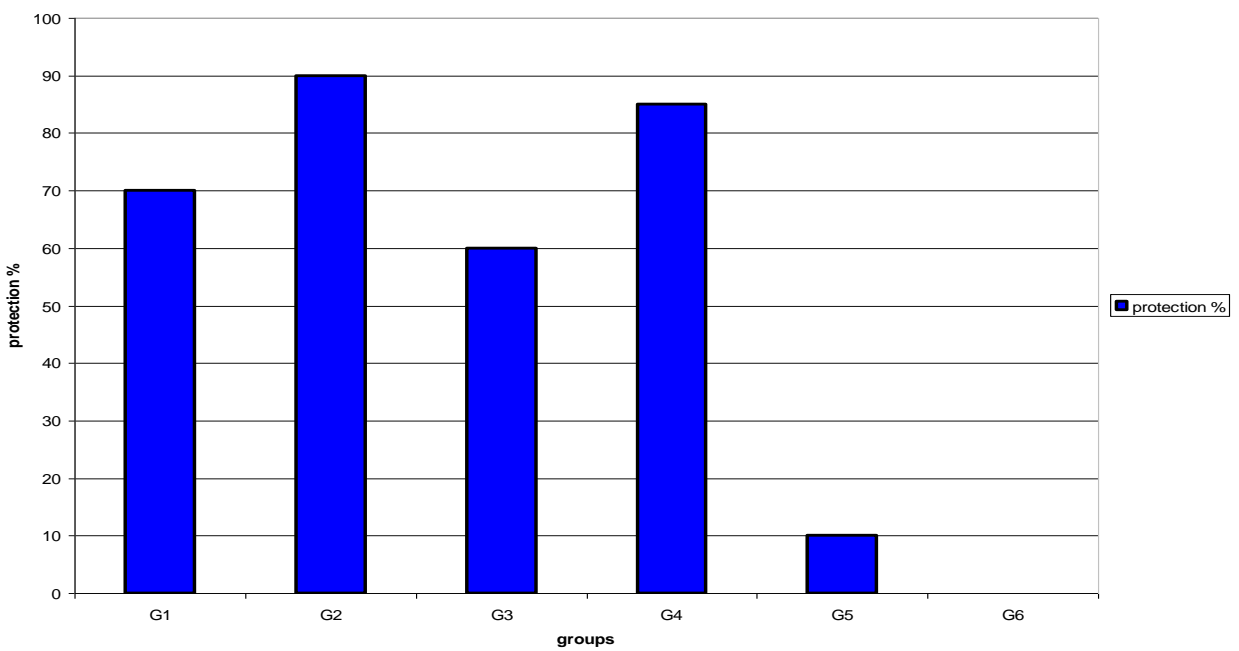

Fig. 3: Mean Antibody ELISA titers at 1-week post challenge of chickens vaccinated with different types of vaccines:

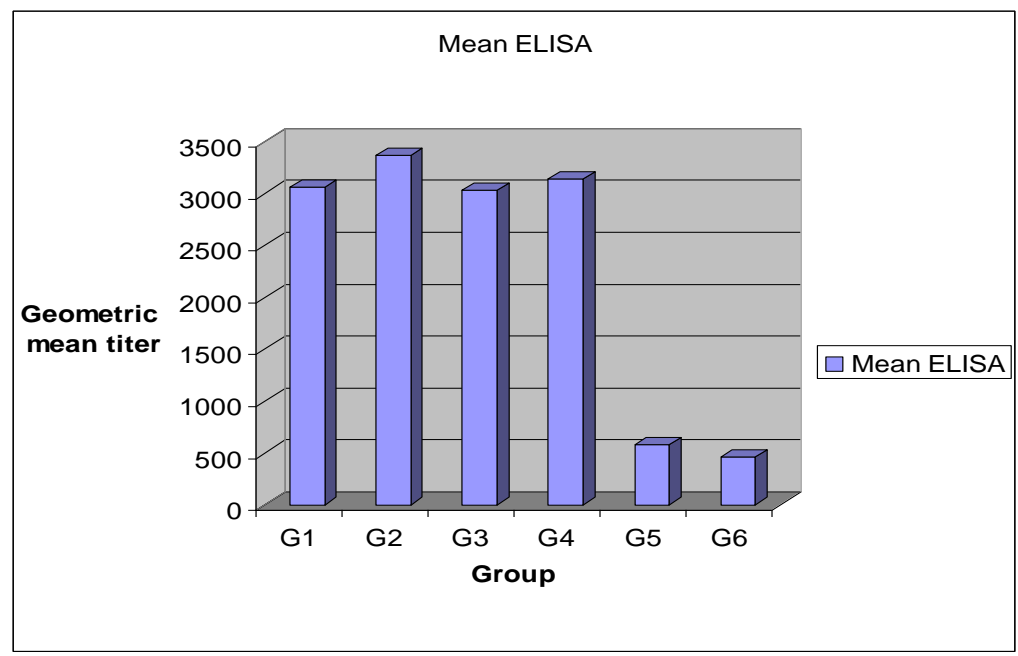


Fig. 4: Comparison of lesions of the bursa of Fabricius after challengeNumber of birds:

Number of birds $\%$

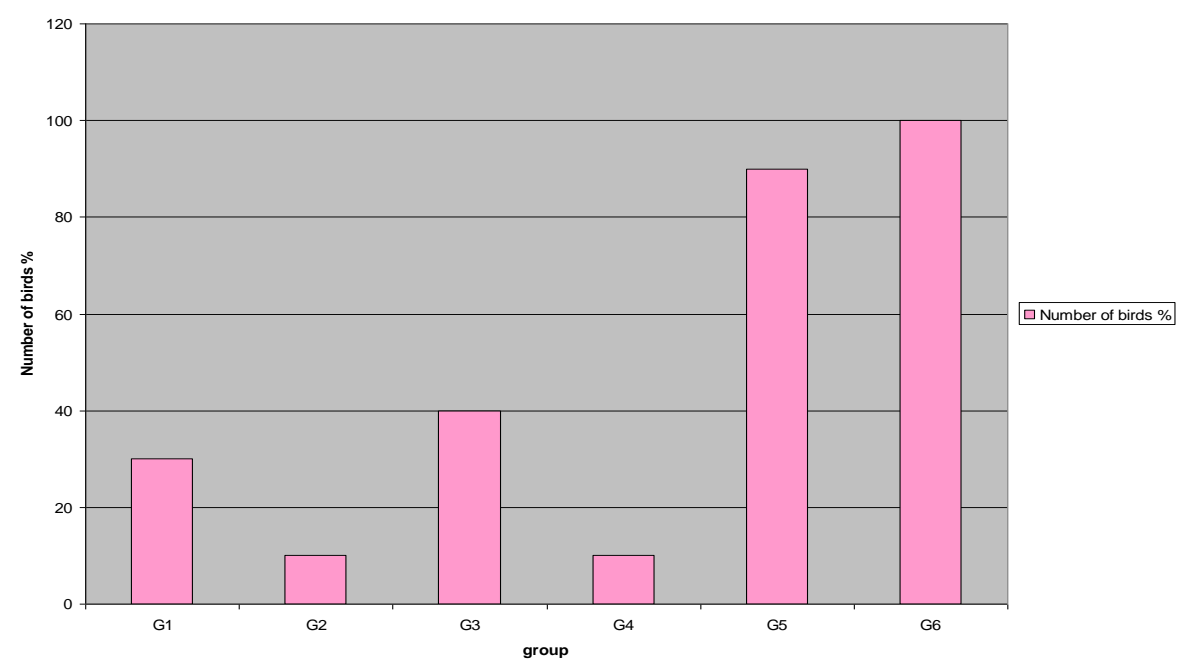

Fig. 5: Comparison of lesions of the bursa of Fabricius after challengeDegree of the bursal lesions:

Degree of lesion \%

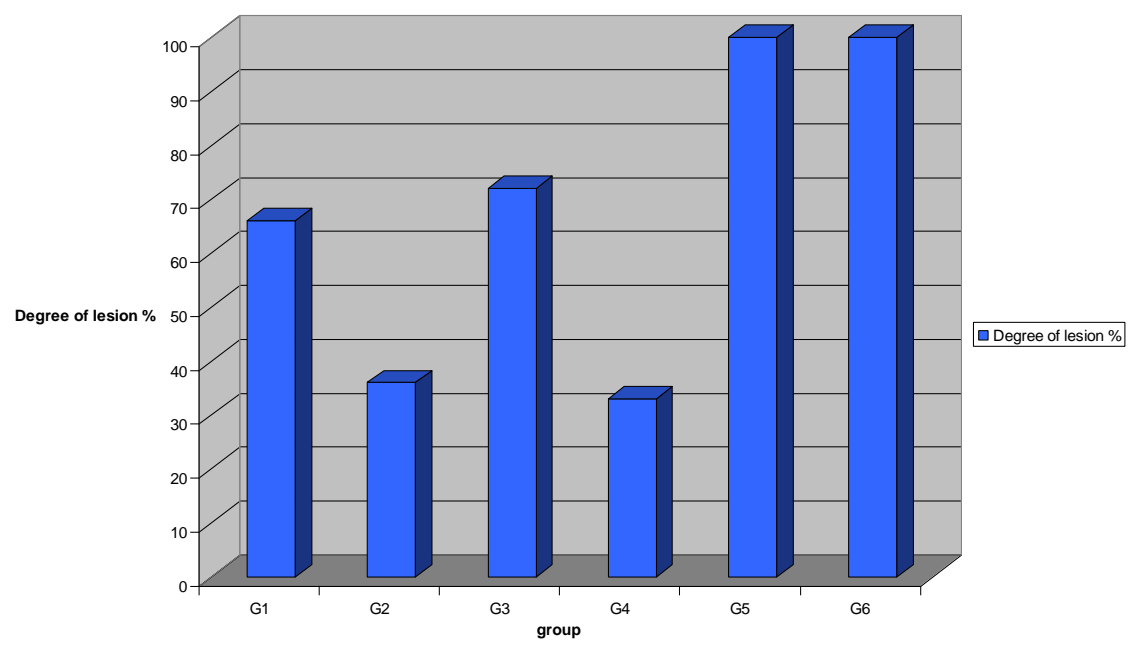




\section{DISCUSSION}

Infectious bursal disease virus (IBDV) remains a serious problem for commercial broiler producers throughout the world. IBD in chickens was first described in 1962 (Cosgrove, 1962). IBDV is a lymphotropic virus able to cause mainly humoral immunosuppresion in chickens infected before three weeks of age (Sharma and Lee, 1983). Vaccination of broiler breeders with live followed by several inactivated vaccines is a common practice in the poultry industry. This practice provides passive immunity to progeny by the transmission of immunoglobulins (IgY) via yolk (van den Berg et al., 1991). In addition, immunity conferred by live vaccines induced mild to moderate bursal atrophy (Tsukamoto et al., 1995).

The efficacy of the in-ovo vaccination was tested with different IBDV vaccines. The vaccine safety-hatchability and first week survival rates are shown in Table (2). The hatchability rate in SPF eggs from group (1) that received vaccine virus of low virulence plus Nigella sativa oil was $80 \%$ while, that of group (3) that vaccinated with the same vaccine without N.sativa oil was $86.66 \%$. Also, the first week mortality rates within hatched chicks in group $(1 \& 3)$ were $4.16 \%$ and $0 \%$, respectively. The chicks hatched from eggs injected with vaccine virus of low virulence remained healthy during the first week observation period after hatch. The previously mentioned results indicated that the inoculation of 18-day old embryonated chicken eggs with IBD vaccine of low virulence did not affect hatchability of eggs seriously or the survival of the hatched chicks. The results agrees with that reported by Sharma (1985) who used the in-ovo vaccination with low virulence IBD vaccines (isolates TC-IBDV and BVM-IBDV).

On the other hand, vaccine virus of moderate virulence was pathogenic for embryos and chickens hatching from eggs with this virus. The data in Table (2) shows that the hatchability percentage in group (2) that inoculated with vaccine virus of moderate virulence with addition of Nigella sativa oil is 53.33 while, that of the vaccine control group (vaccine virus of moderate virulence only) is 60 . Whereas, the first week mortality rate shown in the same table is $25 \%$ and $22.22 \%$ for group 2 and 4, respectively. These results indicated that the IBDV vaccine isolate of moderate virulence that are generally pathogenic for hatched chicks are unsuitable for embryo vaccination. In addition, the results of bursal lesions either macroscopically or microscopically in the inoculated chicks confirmed the observations in Table (2) that the moderate isolate 
was pathogenic for chicks. Sharma evaluated intermediate IBDV vaccines (isolate BV-IBDV and 2512-IBDV) by inoculating 18-day-old embryos and challenging them at 3 weeks of age (Sharma, 1985). He concluded that the vaccine viruses of moderate virulence were not suitable as vaccines in embryos lacking maternal antibodies to IBDV, because they produced lesions in embryos and the vaccinated chicks developed acute IBD after hatching. Efficacy studies with commercial live vaccines in ovo against IBDV caused acute clinical signs of the disease when administered with full dose and hatchability was severely decreased (Sharma et al., 2001). Attempts to administer commercial vaccines in a lower dosage induced less mortality, however, microscopic bursal lesions persisted (Lukert and Saif, 1997). Furthermore, these findings also agree with previous research in SPF and broiler embryos vaccinated with three commercial intermediate vaccines in ovo. The microscopic bursal lesions were observed even when half of the recommended dose was used (Giambrone et al., 2001). Another previous work has indicated that in ovo vaccination against IBDV using live intermediate vaccines, can lead to disease and immunosuppression due to microscopic lesions in the BF (Lukert and Saif, 1997). In addition, these viruses may be able to revert to a virulent state.

However, the percentages of hatched birds and first week survival rates were not significantly different within the Nigella sativa groups even with that of low or moderate isolates of IBDV. As shown in Table (2), the hatchability rate in group (5) that treated with Nigella sative crud oil was $83.33 \%$ and the first week mortality was $4 \%$, while, the same parameters in other Nigella sativa groups (group 1 and 2) were not far away from the other groups without Nigella sativa oil. Theses results indicated that there is no adverse effect of Nigella sativa on the safety-hatchability and the first week survival rates.

The mechanism of protection in embryo-vaccinated chickens is not clear investigated but is probably similar to the mechanism of posthatch vaccination (Sharma, 1985). Thus, injection of IBDV in the prenatal stage did not result in B-cell immunologic tolerance; instead, the virus infected embryos and initiated a protective response. Also, chicken embryos during later stages of development may be capable of responding immunologically to a variety of infectious agents (Sharma, 1985).

The antibody responses as measured by commercial ELISA are shown in Fig. (1) as geometric mean titer. The embryo-vaccinated chickens were examined for antibody sequentially at 1,2 and 3 weeks of 
age. The groups had low levels of antibody at first week of age; at subsequent samplings, the levels of antibodies increased. At these weeks of age all vaccinated groups had significantly higher antibody titers than non-vaccinated ones. The same findings were previously reported by many authors (Sharma, 1985; Jungback et al., 1997 and Giambrone et al., 2001). In addition, Sharma, (1985) mentioned that, the presence of increasing amounts of antibody following embryo vaccination indicated that chickens became infected with the IBD virus and responded immunologically to the virus and did not develop B-cell tolerance.

In this step of work, the effect of Nigella sativa oil as immunostimmulant agent on the vaccinated chickens may be appear at 3 weeks of their age. From Fig. (1), it is clear that the groups that received the Nigella sativa oil with the live vaccine had ELISA titer higher than the other groups that vaccinated with the live vaccines alone. The immunostimmulant effect of Nigella sativa was noticed and reviewed previously by many researchers (Hanafy and Hatem, 1991; El-Kadi and Kandil 1986; Haq et al., 1995; Osman, and EL-Barody, 1999 and Swamy and Tan, 2000).

The embryo-vaccinated chickens were examined for protection against challenge with virulent IBDV. All vaccinated groups showed protection against IBDV in comparison to that non vaccinated with any of IBDV vaccine (negative control or treated with Nigella sativa only). The results of the IBDV challenge studies are demonstrated in figures (2, 3,4 and 5). The vaccinated groups 1,2, 3 and 4 showed comparable protection. The percentages of protection exhibited in Fig (2) correlated well with the mean ELISA antibody titers shown in Fig (3) that measured one-week post-challenge. In chickens vaccinated with moderate isolate vaccine, the protection was higher than those vaccinated with low virulence one. There was no significantly difference between groups vaccinated with vaccine plus Nigella sativa and that received the vaccine only either of low or moderate virulence nature. By comparing the number of birds that exhibited bursal lesions after challenge within the studied vaccinated groups (Fig. 4), it was found that the higher number was among the groups that vaccinated with vaccine virus of low virulence in spite the control groups. Furthermore, the percentages of bursl lesion degree shown in Fig. (5) revealed that the vaccine virus of moderate virulence protect the challenged birds than the low one. Thus, the previously results revealed that the embryovaccination protected chickens against challenge with virulent IBDV at first weeks of ages which is similar to that reported by Sharma, (1985) 
but he also mentioned that the embryo-vaccination protection was not better than the protection one week after vaccination at hatch.

In conclusion, we have noted that the vaccination of specificpathogen free chickens as embryos with IBDV alone or in combination with Nigella sativa oil resulted in initiation of immune response in hatched chickens. All chickens hatched from vaccine-injected eggs had neutralizing antibodies at early days of age and were protected against challenge with pathogenic IBDV. Finally, the IBD vaccines especially of low virulence nature could be safely combined with Nigella sativa oil and used in the in-ovo route of vaccination.

\section{REFERENCES}

Cosgrove, A.S. (1962): An apparently new disease of chickens-avian nephrosis. Avian Dis., 6: 385-389.

EL-Alfy, T.S.; EL-Fatatry and Toama, M.A. (1975): Isolation and assignment of an antimicrobial principle from the volatile of Niglla sativa seeds. Phamazia, 30: 109-111.

El-Kadi, A. and Kandil, O. (1986): Effect of Nigella sativa (the black seed) on immunity. Proceeding of the $4^{\text {th }}$ International Conference on Islamic Medicine, Kuwait. BullIslamic Med. 4: 344-8.

Giambrone, J.J.; Dormitorio, T. and Brown, T. (2001): Safety and efficacy of in ovo administration of infectious bursal disease viral vaccines. Avian Dis., 45: 144-148.

Hanafy, M.S. and Hatem, M.E. (1991): Studies on the antimicrobial activity of Nigella sativa seed (black cumin). J. Ethnopharmacol. 1991 Sep; 34: 275-8 1795532 (P,S,G,E,B).

Haq, A.; Abdullatif, M.; Lobo, P.I.; Khabar, K.S.; Sheth, K.V. and AlSedairy, S.T. (1995): Nigella sativa: Effect on human lympocytes and polymorphonuclear leucocyte phagocytic activity. Immunopharmacology, 30 (2): 147-50.

Houghton, P.J.; Zarka, R.; Delas, H.B. and Hoult, J.R.S. (1995): Fixed oil of Niglla sativa and derived thymoquinone lipid peroxidation. Planta Medico., 61: 33-36.

Jungbach, C.; Kuchler, B.; Finkler, H.; Frank, A.; Heer, A.; Schirk, E. $M$. and Klimek, J. (1997): In-ovo application of IBD vaccines. A comparison of efficacy after in ovo application and vaccination of one day old chickens. In Proceedings of the WPSA-Congress, Budapest. Hungeria. p 272-278. 
Kibenge, F.S.; Dhillon, A.S. and Russell, R.G. (1988): "Biochemistry and immunology of infectious bursal disease virus" J. Gen. Virol., 69: 1757-1775.

Lukert, P.D. and Saif, Y.M. (1997): Infectious bursal disease virus. In Disease of Poultry, (B.W..Calnek, et al., ed.) $10^{\text {th }}$ Ed., pp 721738. Iowa State University Press, Ames Iowa.

Lutticken, D.; Van Loon, A.A.W.M. and de Vries, M.J.H.S. (1994): "Determination of the breakthrough titre of IBD vaccines or IBD challenge strains in MDA+ chickens". In Proceedings of the International Symposium on Infectious Bursal Disease and Chicken Infectious Anaemia. Rauischholzhausen, Germany, $\mathrm{p}$ 272-278.

Mahfouz, M. and EL-Dakhakhny, M. (1960): Some chemical and pharmacological properties of the new anti asthmatic drug Nigellone. Egypt. Pharm. Bull., 24: 411-424.

Mandour, A.A.; Ashry, K.M. and Hedaya, S.A. (1998): Biochemical profile of serum constituents of broiler chickens supplemented with different levels of Niglla sativa with special references to its effect on hormonal and mineral concentrations. Egypt. Poult. Sci., 18: 429-439.

Nunoya, T.; Otaki, Y.; Tajima, M.; Hiraga, M. and Saito, T. (1992): "Occurrence of acute infectious bursal disease with high mortality in Japan and pathogenicity of field isolates in specific-pathogen-free chickens". Avian Dis., 36: 597-609.

Osman, A.M.A. and EL-Barody, M.A.A. (1999): Growth performance and immune response of broiler chicks as affected by diets density and Niglla sativa seeds supplementation. Egypt. Poult. Sci., 19: 619-633.

Sharma, J.M. (1985): Embryo vaccination with IBDV alone or in combination with Marek's disease vaccine. Avian Dis. 29: 1155-1169.

Sharma, J.M. and Burmester, B.R. (1982): "Resistance to Marek's disease at hatching in chickens vaccinated as embryos with the turkey herpesvirus". Avian Dis., 26:134-149.

Sharma, J.M. and Lee, L.F. (1983): "Effect of infectious bursal disease on natural killer cell activity and mitogenic response of chicken lymphoid cells: role of adherent cells in cellular immune suppression". Infect. Immun., 42: 747-754. 
Sharma, J.M.; Zhang, Y.; Jensen, D.; Rautenschlein, S. and Yeh, H.Y. (2001): Field trial in commercial broilers with a multivalent in ovo vaccine comprising a mixture of live viral vaccines against Marek's disease, infectious bursal disease, Newcastle disease, and Fowl pox. Avian Dis., 46: 613-622.

Swamy, S.M.K. and Tan, B.K.H. (2000): Cytotoxic and immunopotentiating effects of ethanolic extract of Nigella sativa L. seeds. J. Ethnopharmacol. 70, 1.7.

Tsukamoto, K.; Tanimura, N.; Kakita, S.; Ota, K.; Mase, M.; Imai, K. and Hibara, H. (1995): "Efficacy of three live vaccines against highly virulent infectious bursal disease virus and the optimum vaccination". Avian Dis., 39: 218-229.

Van den Berg, T.P.; Gonze, M. and Meulemans, G. (1991): "Acute infectious bursal disease virus in poultry: isolation and characterization of a highly virulent strains". Avian Pathol., 20: 133-143. 